\title{
Connectivity in Two-Dimensional Lattice Networks
}

\author{
Lei Zhang, Lin Cai, Jianping Pan \\ University of Victoria, Victoria, BC, Canada
}

\begin{abstract}
Connectivity has been extensively studied in ad hoc networks, most recently with the application of percolation theory in two-dimensional square lattices. Given a message source and the bond probability to connect neighbor vertexes on the lattice, percolation theory tries to determine the critical bond probability above which there exists an infinite connected giant component with high probability. This paper studies a related but different problem: what is the connectivity from the source to any vertex on the square lattice following certain directions? The original directed percolation problem has been studied in statistical physics for more than half a century, with only simulation results available. In this paper, by using a recursive decomposition approach, we have obtained the analytical expressions for directed connectivity. The results can be widely used in wireless and mobile ad hoc networks, including vehicular ad hoc networks.
\end{abstract}

Index Terms-Connectivity, square lattice, directed percolation

\section{INTRODUCTION}

Connectivity has been extensively studied in ad hoc networks [1]-[5] where a group of nodes are distributed in the space without any communication infrastructures. To deliver a message to its destination, multi-hop wireless communications and/or short-range contacts are needed. Connectivity is defined as the probability to deliver the message to the destination at a certain time or within a time duration (e.g., with storecarry-and-forward). Such scenarios are termed as wireless, mobile, or vehicular ad hoc networks (W/M/VANET) [6]-[8] or delay/disruption-tolerant networks (DTN).

The study of connectivity in two-dimensional (2D) ad hoc networks has attracted lots of attention in the community, most recently with geometrical probability, stochastic geometry, and percolation theories [9]-[11]. When the network can be adequately modeled as a 2D square lattice (e.g., VANET in city blocks), percolation theory has been widely used. Initially in statistical physics, percolation theory studies the process of liquid filtering through porous materials. In bond percolation, a fundamental problem is to determine the critical bond probability to connect each two neighbor vertexes. When the bond probability is above the critical value, there exists an infinite giant component (and an infinite number of finite components) with high probability [12]. If the filtering directions are given, it is called directed percolation (DP).

In this paper, we study a related but different problem: directed connectivity (DC), i.e., given a message source and the bond probability to connect neighbor vertexes on a square lattice, what is the probability for the message to reach an arbitrary vertex following certain directions? The problem appears to be similar to DP at the macroscopic level, but different microscopically as DP only cares about the existence of a giant component, while DC has to determine the connectivity to each vertex. DC is more relevant to network connectivity.

Despite the effort in more than half a century, DP and many related problems are mainly solved numerically by simulation. The most related work determined the critical probability analytically of a square lattice where the vertical bond probability is $p_{y}$ and the horizontal probabilities are 1 and $p_{x}$ interleaved at different layers [13]. Conceptually, DC problems are even harder than DP. However, by extending our previous work on 2D ladder connectivity [8] and by using a new recursive decomposition approach, we have obtained the analytical expression for the DC problem on square lattices. The approach shall extend to lattices with different horizontal and vertical bond probabilities and arbitrary shape.

The contributions of this paper are summarized as follows. First, to the best of our knowledge, this is the first time that the DC problem is solved analytically on square lattices, which can quickly determine the network connectivity without lengthy simulation. Even though the results are based on square lattices, they can offer some insights when clustering and aggregation are possible in full 2D networks. Second, as DC problems are highly related to DP problems, we can also use the DC results to predict the behavior of DP problems.

The remainder of this paper is organized as follows. In Section II, we review the most related work in directed percolation and its application in ad hoc networks. We present our analysis framework and derivation in Section III, and evaluate the efficacy of the analytical expression in Section IV. Section V offers further discussion and the application of these results in ad hoc networks, particularly VANET, and Section VI concludes the paper with future work.

\section{RELATED WORK}

\section{A. Directed Percolation}

Percolation has been observed in many natural and manmade systems, initially motivated by the process of liquid filtering through porous materials [12]. The process can be modeled by vertexes (sites) and edges (bonds) in certain dimensions, and depending on whether to occupy a site or bond, two processes are defined: site or bond percolation. Also a site percolation can be converted into a bond percolation (not always vice versa). In a homogeneous bond percolation, the bond probability $p \in[0,1]$ connecting two neighbor vertexes (e.g., the liquid filters through the bond) is considered. Assuming an infinite number of vertexes and edges, percolation occurs when there exists an infinite connected giant component (and an infinite number of finite components). Percolation is more likely to occur with a larger $p$, so when $p$ reduces from 1 
to 0 , percolation either occurs or not, exhibiting a sharp phase transition at the so-called critical probability $p_{c}$.

A rich set of research outcome has appeared for various percolation scenarios in different dimensions. Depending on whether the bonding between neighbor vertexes is directional or not, percolation can be further classified into (isotropic) percolation and directed percolation (DP). Although mostly by numerical approaches, the critical probabilities for many lattice (discrete) and continuum models have been found or approximated [14]. For example, for 2D square lattices, $p_{c}$ is 0.5 by proof for bond percolation, and 0.59 by approximation for site percolation. For other 2D regular tiling, both the bond and site percolation thresholds can be determined for triangles, but only the bond percolation threshold for hexagons. However, the directed version of them turns out to be much harder and only numerical results are available, even for regular triangles, squares and hexagons. Besides critical probabilities, the convergence behaviors around $p_{c}$ are also heavily investigated.

The most related work is the directed percolation on a square lattice where the vertical (or one of the two dimensions) bond probability is $p_{y}$ and the horizontal (i.e., the other dimension) bond probabilities are 1 and $p_{x}$ interleaved at different layers [13]. By defining the "wet" and "primary wet" edges on each layer, the critical probability is found analytically when the connectivity from the origin to the farthest vertex in a finite square lattice of a given aspect ratio $\alpha$ is transitioning from 0 to 0.5 and to 1 . When $p_{x}=1$, this model degenerates into a quasi-2D model with results known long ago. Similarly, when $p_{x}=0$, it degenerates into the quasi model with vertical bond probability $p_{y}^{2}$. Most recently, the author also discussed the convergence behaviors of such an interleaved model [15].

\section{B. Connectivity in Ad Hoc Networks}

On the other hand, connectivity has also been extensively studied in ad hoc networks, mostly in the 2D Euclidean spaces [1]-[5]. Without fixed communication infrastructures, nodes in ad hoc networks have to rely on their neighbors or leverage the mobility of them to deliver messages to the destination, often in a multi-hop manner through wireless communications and/or short-range contacts, so the connectivity has to be characterized probabilistically. A wide variety of ad hoc networks exist, ranging from stationary (sensor networks [16]) to mobile ones. VANET is a special type of the latter, where vehicles are involved as the communication source, destination and relay [6]-[8]. High vehicle velocity introduces more challenges to connectivity, but the predictable mobility also offers new opportunities. For example, along a highway, vehicles travel in one dimension, possibly also communicating with the vehicles on the reverse direction. In a city block scenario, a 2D square lattice is often used to approximate the road grid.

Analytical and algorithmic tools in graph theory and computational geometry have been widely used in the modeling and analysis of connectivity in ad hoc networks [17], together with geometrical probability, stochastic geometry, and percolation theories in recent years. For example, a connected dominating set is introduced in ad hoc networks to create a virtual backbone for the network [18]. Geometrical probability tools offer the characterization of distance distributions among nodes in and between different geometry shapes (e.g., triangles, rectangles and hexagons [19]-[21]), and stochastic geometry tools further introduce the time line in the random process of node coverage and connectivity [22]. Additional nodes can be deployed, some even mobile, to improve the connectivity.

Most recently, percolation theory has found a wide range of applications in networking research, particularly on the connectivity in ad hoc networks [23]-[25]. Many networking scenarios can be adequately modeled as percolation on square lattices, either individually (e.g., VANET in city blocks) or after clustering and aggregation (cluster heads in wireless sensor networks). Although square lattices are most widely used, other 2D regular tilings can also be used (e.g., hexagons for cellular systems and rhombuses or triangles for cells with directional antennas). For messages with a given destination, or vehicles traveling in certain directions, geographical forwarding is often used to minimize the network overhead due to flooding [8]. Thus directed percolation becomes a premier model in such scenarios, and most existing work applies the results from isotropic or directed percolation on square lattices.

This paper studies the directed connectivity (DC) problem on square lattices, motivated by the VANET connectivity in city scenarios. We first try to establish the analytical expression for the directed connectivity from a given message source to any possible destinations in the network. This problem is related to DP but more microscopic, as DP is only concerned about the existence of an infinite giant component, but the coexistence of an infinite number of finite components also indicates (although not characterizing quantitatively) that some destinations are not connected. To the best of our knowledge, this is the first analytical result in the literature, other than our previous work on a special case (i.e., 2D ladders [8]). Although we assume an identical bond probability in this paper, our work can be easily extended to more general DC problems with variable bond probabilities. With the analytical results on the DC problem, we also hope to shed some new light on the half-century old DP problem.

\section{ANALYSIS FRAMEWORK}

In this section, we first give the system model for directed connectivity, and then present the analytical framework and derivation results for $1 * 1,2 * 2$ to $m * n$ lattices.

\section{A. System Model}

As shown in Fig. 1(a), we consider a 2D lattice $L(m, n)$, with edges parallel to the $x$ and $y$ axis for notation convenience. A message is generated at the origin $O=(0,0)$ at time $t=0$, and propagated along the lattice edges in the directions indicated by arrows. Assuming the bond probability $p$ of any two neighbor vertexes, what we want to know is the connectivity probability from the origin to $(m, n)$, as a 

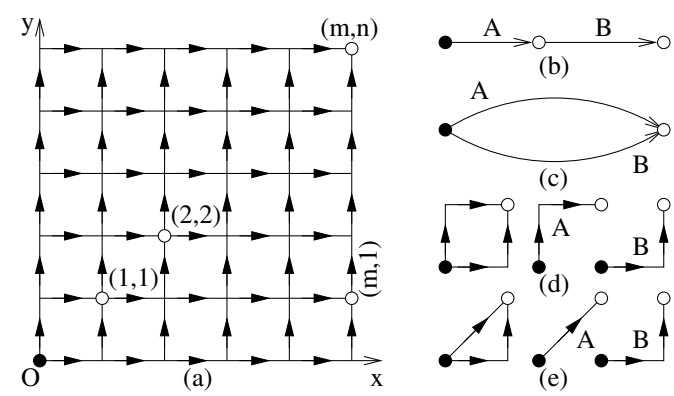

Fig. 1: System model and basic principles.

function of $p, m$ and $n$. In Section $\mathrm{V}$, we will discuss how to calculate different $p$ in different scenarios, e.g., VANET in city blocks, in lattice networks with different horizontal and vertical bond probability $p_{x}$ and $p_{y}$, and when the lattice is not necessary square and the bond probability is arbitrary.

Even with such a simplified model, deriving the connectivity probability at $(m, n)$, denoted as $P(m, n)$, is still a hard problem. For example, to reach $(m, n)$, the message can go through $(m-1, n)$ or $(m, n-1)$ as the last hop. However, even if $P(m-1, n)$ and $P(m, n-1)$ were known, it is still very difficult to derive $P(m, n)$, as the paths from $(0,0)$ are not independent before they reach the last hop. A brute-force approach has to enumerate all possible paths and overlapping (i.e., when different paths share the same edges) and suffer the combinatorial explosion problem on the exponent. This is also the reason why DP remains unsolved for so many years.

To facilitate the presentation, we also illustrate some basic principles and simple cases in Fig. 1. First, if there are two directed paths $A$ and $B$ connected by a common vertex serially as shown in Fig. 1(b), the end-to-end connectivity is $P(A B)=$ $P(A) P(B)$, as $A$ and $B$ are always independent with directed edges. Here, we define $P(A)$ and $P(B)$ as the probabilities that path $A$ and $B$ are connected, respectively. Second, if there are two parallel paths $A$ and $B$ connecting the source and destination as shown in Fig. 1(c), the source-to-destination connectivity is $P(A+B)=P(A)+P(B)-P(A B)$ according to the principle of inclusion and exclusion (PIE). These two principles can be used to solve the $1 * 1$ lattice problem as shown in Fig. 1(d): $P(A)=P(B)=p \cdot p=p^{2}$, and $P(1,1)=P(A)+P(B)-P(A) P(B)=2 p^{2}-p^{4}$ as $A$ and $B$ are independent but not mutually exclusive. Later we will find that we also encounter a triangular grid as shown in Fig. 1(e), and the end-to-end connectivity in this case is $P_{T}(1,1)=p+p^{2}-p \cdot p^{2}=p+p^{2}-p^{3}$. The cases become more complicated when $A$ and $B$ are also dependent.

\section{B. $2 * 2$ Lattices}

Following the same principles, we attempt to solve the $2 * 2$ lattice problem as shown in Fig. 2. Similarly, the top-leftmost path $A$ and the union of all other paths, $\mathcal{B}$, are identified. But the difference from Section III-A is that they are no longer independent (as $A$ and $\mathcal{B}$ have many overlapping edges). A naive approach is to consider each edge along $A$ separately and check its impact on $\mathcal{B}$, but depending on the edge connected or not, there are $2^{4}$ cases of a single path $A$ (and more cases

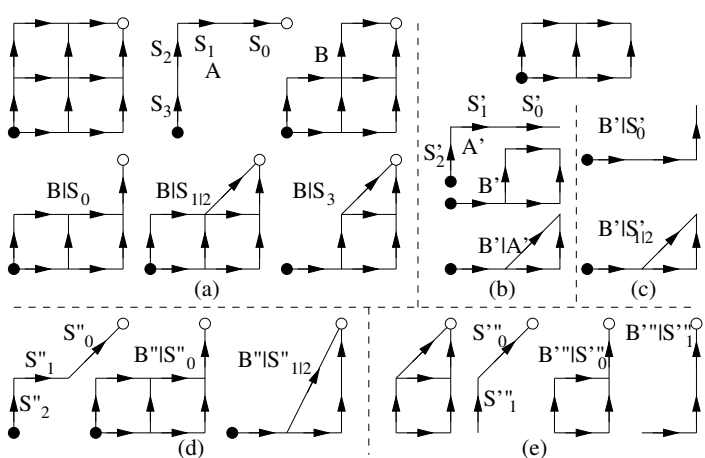

Fig. 2: The decomposition of a $2 * 2$ lattice.

introduced in $\mathcal{B}$ ). If using the PIE principle, after the first level of decomposition, $\mathcal{B}$ with more than two layers is very difficult if not impossible to be decomposed further. Because most edges, other than the bottom-rightmost ones, are shared by many paths. This also renders our previous approach [8] on ladders of one layer not applicable to lattices. Observing $A$ is a single path (i.e., no branches possible), we can have a simple partition. As shown in Fig. 2(a), define $S_{i}$ as the event that the last $i$ edges along $A$ leading to the destination are all connected, but the last $(i+1)$-th one is not, so $P\left(S_{i}\right)=p^{i}(1-$ $p)$ for $0 \leq i \leq m+n-1$. For the origin and destination being connected, we then have $m+n+1$ mutually exclusive cases, including $\mathcal{B} \mid S_{i}$ and $A$ being connected where $P(A)=p^{m+n}$. Define the probability that $\mathcal{B}$ is connected given $S_{i}$ as $P\left(\mathcal{B} \mid S_{i}\right)$, we have

$$
\begin{aligned}
P(m, n) & =P(A+\mathcal{B})=1-P(\overline{\mathcal{B}+A}) \\
& =1-P(\overline{\mathcal{B}} \bar{A}) \\
& =1-P\left(\overline{\mathcal{B}} \bigcup_{i=0}^{m+n-1} S_{i}\right) \\
& =1-\sum_{i=0}^{m+n-1} P\left(\overline{\mathcal{B}} \mid S_{i}\right) P\left(S_{i}\right) \\
& =1-\sum_{i=0}^{m+n-1}\left(1-P\left(\mathcal{B} \mid S_{i}\right)\right) P\left(S_{i}\right) \\
& =P(A)+\sum_{i=0}^{m+n-1} P\left(\mathcal{B} \mid S_{i}\right) P\left(S_{i}\right),
\end{aligned}
$$

where (1) is due to De Morgan's law, (2) due to $\bigcup_{i=0}^{m+n-1} S_{i}=$ $\bar{A}$, (3) due to $S_{i}$ being mutually exclusive, and (4) due to $\sum_{i=0}^{m+n-1} P\left(S_{i}\right)=P(\bar{A})=1-P(A)$, i.e., $A$ and $S_{i}$ partition and constitute the entire event space in total probability.

For $L(2,2)$, given $S_{0}$, no connection is possible if the message reaches $(0,2)$ or $(1,2)$, so we can remove the edges adjacent to them and have $\mathcal{B} \mid S_{0}$ as shown in Fig. 2(a). Given $S_{1}$, it implies that $(1,2)$ and $(2,2)$ are connected, but no connection is possible through $(0,2)$, so we can merge $(1,2)$ with $(2,2)$, and remove the edges adjacent to $(0,2)$. Since $S_{1}$ and $S_{2}$ have the same effect, they are illustrated as $\mathcal{B} \mid S_{1 \mid 2}$ in Fig. 2(a). Given $S_{3}$, it implies that $(0,1),(0,2),(1,2)$ and $(2,2)$ are connected sequentially, so they can be merged; no 

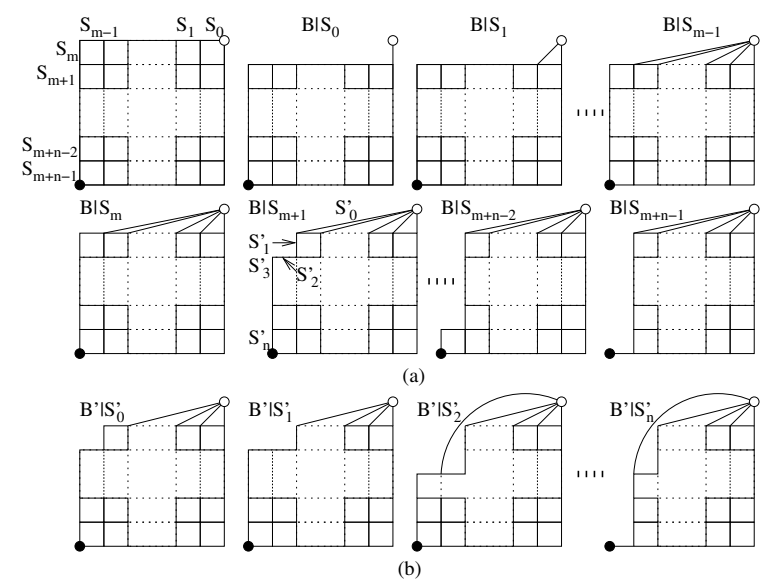

Fig. 3: The decomposition of an $m * n$ lattice.

connection is possible through $(0,1)$, so the edges adjacent to it have to be removed, as $\mathcal{B} \mid S_{3}$ in Fig. 2(a).

After this decomposition, we have $\mathcal{B} \mid S_{0 . .3}$. Using the serial principle, $B \mid S_{0}$ can be decomposed into a $2 * 1$ lattice (or ladder) and an edge. Figure 2(b) shows how we further decompose the ladder into $A^{\prime}, \mathcal{B}^{\prime}$ and $\mathcal{B}^{\prime} \mid A^{\prime}$ following the conditional probability approach we previously proposed for ladders specifically [8], while Fig. 2(c) shows the new total probability approach with $\mathcal{B}^{\prime} \mid S_{0 . .2}^{\prime}$, which can both be solved directly using the serial principle, $P(1,1)$ and $P_{T}(1,1)$ : the results are the same, but the new approach is simpler, especially when we have higher-order lattices. Similarly for $\mathcal{B} \mid S_{1 \mid 2}$, they are decomposed in Fig. 2(d) to components of known connectivity (e.g., $\mathcal{B} " \mid S{ }^{\prime}{ }_{0}$ is the same as $\mathcal{B} \mid S_{1 \mid 2}$ ), and part of $\mathcal{B} \mid S_{3}$ is decomposed in Fig. 2(e), where the serial principle and $P(1,1)$ can be applied. Using the total probability approach, the decomposed components can be reassembled, so is the connectivity, and $P(2,2)$ can be recovered as follows

$$
\begin{aligned}
P(A) & =p^{4} \\
P\left(\mathcal{B} \mid S_{0}\right)= & p^{8}-p^{7}-2 p^{6}+3 p^{4} \\
P\left(\mathcal{B} \mid S_{1 \mid 2}\right)= & -p^{9}+3 p^{8}-3 p^{6}-3 p^{5}+3 p^{4}+2 p^{3} \\
P\left(\mathcal{B} \mid S_{3}\right)= & p^{7}-2 p^{6}-p^{5}+2 p^{4}+p^{3} \\
P(2,2)= & P(A)+\sum_{i=0}^{3} P\left(\mathcal{B} \mid S_{i}\right) P\left(S_{i}\right) \\
= & p^{12}-4 p^{11}+2 p^{10}+4 p^{9} \\
& +2 p^{8}-4 p^{7}-6 p^{6}+6 p^{4}
\end{aligned}
$$

\section{C. $m * n$ Lattices}

Following the new total probability approach, we attempt to solve the generic $m * n$ lattice problem as shown in Fig. 3(a). For clarify, we have omitted the arrow on edges in the following figures. Similar to $\mathcal{B} \mid S_{i}$ in Section III-B, we can first remove the edges or merge the vertexes on the top row of an $m * n$ lattice, as $\mathcal{B} \mid S_{0 . . m-1}$, and then remove the edges or merge the vertexes along the leftmost column of the lattice, as $\mathcal{B} \mid S_{m . . m+n-1}$, by considering the top-leftmost path $A$ and events $S_{0 . . m+n-1}$, as well as their impacts on $\mathcal{B}$.
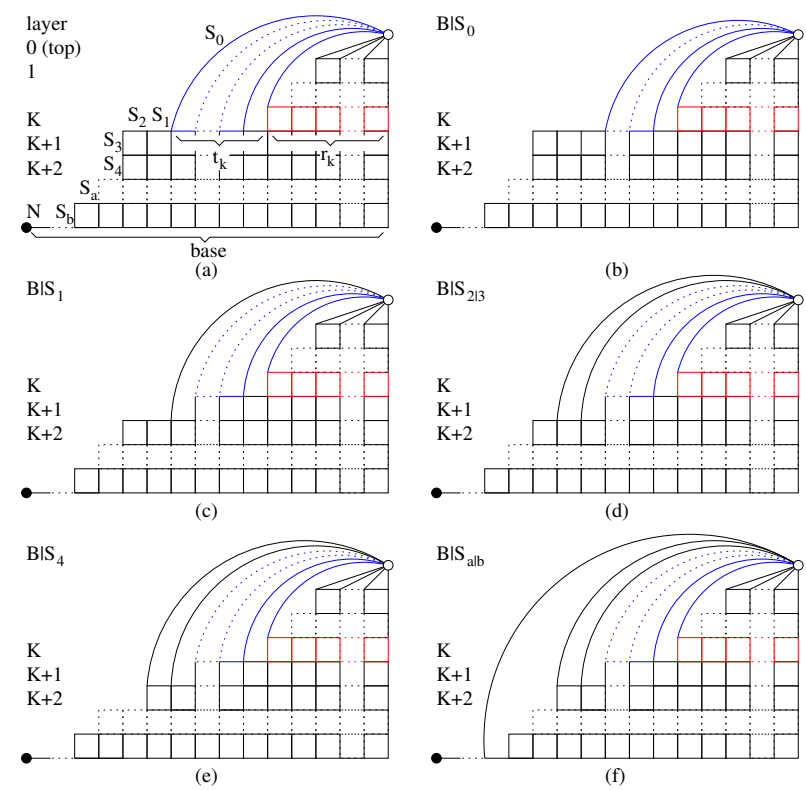

Fig. 4: The decomposition of a Tower.

After this decomposition, similar to Section III-B, we need to further decompose the components eventually to the ones of known connectivity. For example, $\mathcal{B} \mid S_{0}$ contains an $m *(n-1)$ lattice and an edge, which leads to a recursion among lattices. $\mathcal{B} \mid S_{m+1}$ is further decomposed in Fig. 3(b) and similarly for all the other $\mathcal{B} \mid S_{i}$. Although all $\mathcal{B} \mid S_{i}$ s and their decomposed components have different structures, we have found the similarities between these structures during the decomposition process, and we can introduce a generic structure called Tower to formulate the recursions among them.

Figure 4(a) shows the generic structure of Tower $\mathcal{T}$. All the decomposed components of the $m * n$ lattice, plus the lattice itself, can be regarded as a special case of $\mathcal{T} . \mathcal{T}$ has a layered structure, with more blocks near the bottom, as we remove edges and merge vertexes gradually along the top-leftmost portion of the tower. The source is the bottomleftmost vertex at $(0,0)$, and the destination is top-rightmost at $(m, n)$. On each layer, there are two types of building blocks: triangles and rectangles. Each triangle $\triangle$, highlighted in blue on layer $K$ in Fig. 4, is composed of two direct shortcuts to $(m, n)$ and one or two ordinary lattice edges. A rectangle $\square$, highlighted in red, is the block originally in the lattice and not affected by the decomposition process yet. Depending on the number $\left(t_{i}\right)$ of $\triangle \mathrm{s}$ and that $\left(r_{i}\right)$ of $\square \mathrm{s}$, we can denote layer $i$ by $\left(t_{i}, r_{i}\right)$, except for the base $b$ which is represented by the number of the bottom edges. Taking into account all the layers in a configuration for the tower, we can denote it by $\mathcal{T}\left(\left(t_{0}, 0\right), \sqcup_{i=1}^{K}\left(t_{i}, r_{i}\right), \sqcup_{i=K+1}^{N}\left(0, r_{i}\right), b\right)$, where $\sqcup$ represents a series of layers. Be aware that we distinguish four types of layers: 1) the top layer 0 with $\triangle \mathrm{s}$ only; 2) the mixed ones of both $\triangle \mathrm{s}$ and $\square \mathrm{s}$ from layer 1 to $K$; 3) the ones with $\square$ s only from layer $K+1$ to $N$; 4) the base $b$. For example, $\mathcal{T}\left(\sqcup_{i=0}^{n-1}(0, m), m\right)$ is the original $m * n$ lattice, $\mathcal{T}\left((1,0), \sqcup_{i=1}^{n-1}(0, m), m\right)$ is the $\mathcal{B} \mid S_{1}$ in Fig. 3, $\mathcal{T}\left((m-1,0),(0, m-1), \sqcup_{i=2}^{n-1}(0, m), m\right)$ is $\mathcal{B} \mid S_{m+1}$, and 
$\left.\mathcal{T}\left((m-1,0), \sqcup_{i=1}^{n-1}(0, m-1), m\right)\right)$ is $\mathcal{B} \mid S_{m+n-1}$.

For a generic tower as shown in Fig. 4, we can identify the top-leftmost path as $A$ and a series of events $S_{i}$. Recall that $S_{i}$ means the last $i$ edges along $A$ leading to $(m, n)$, including the original lattice edges (either horizontal or vertical) and shortcut edges, are connected, but the last $(i+1)$-th one is not. With a bit abuse of notation, let $s_{i}$ represent the last broken edge toward $(m, n)$ and the edge $s_{i}$ corresponds to the event $S_{i}$. It is important to recall that the decomposition happens serially from event $S_{0}$ to $S_{b}$, corresponding to Fig. 4(a). Each decomposition (e.g., $S_{i}$ 's) is performed on the tower obtained from the previous decomposition (i.e., $S_{i-1}$ 's). Essentially we have four types of edges along $A: 1)$ one shortcut edge (e.g., $s_{0}$ in Fig. 4(a)) on layer $K$; 2) horizontal edges (e.g., $s_{1}$ and $s_{2}$ ), of which there are $r_{i+1}-t_{i}-r_{i}$ on each layer for $i>=K ; 3$ ) at most one vertical and topmost corner edge $\left(s_{3}\right.$ or $s_{a}$ or $\left.s_{b}\right)$ on each layer for $i>K$ and $r_{i}>t_{i-1}+r_{i-1}$; 4) at most one vertical but not topmost edges $\left(s_{4}\right)$ on each layer for $i>K$ and $r_{i}=r_{i-1}$. In the following, we will show how each type of $S_{i}$ can reduce a tower to another of less complexity.

1) The shortcut edge along $A$ : For example, $s_{0}$ is in $A$ but not in $\mathcal{B}$, so whether it is broken or not does not affect $\mathcal{B}$, and $\mathcal{B} \mid S_{0}=\mathcal{B}$ as shown in Fig. 4(b). Using the tower notation, $\mathcal{T}\left(\cdots,\left(t_{K}, r_{K}\right), \cdots\right) \stackrel{S_{0}}{\longrightarrow} \mathcal{T}^{\prime}\left(\cdots,\left(t_{K}-1, r_{K}\right), \cdots\right)$ with the absence of the shortcut edge at layer $K$, and no changes in other layers, so the tower complexity is reduced.

2) The horizontal edges along $A$ : For $s_{1}$, it is in both $A$ and $\mathcal{B}$, and if it is broken but $s_{0}$ is connected, it will remove all the horizontal edges left to it on layer $K$ and introduce a shortcut to $(m, n)$ directly on layer $K+1$, as shown in Fig. 4(c). Using the tower notation, $\mathcal{T}\left(\cdots,\left(t_{K}, r_{K}\right),\left(0, r_{K+1}\right), \cdots\right) \stackrel{S_{1}}{\longrightarrow}$ $\mathcal{T}^{\prime}\left(\cdots,\left(t_{K}-1, r_{K}\right),\left(1, t_{K}+r_{K}-1\right), \cdots\right)$, i.e., one $\triangle$ on layer $K$ is removed, but one $\triangle$ on layer $K+1$ is introduced. However, the number of $\square$ s on layer $K+1$ has been reduced to $r_{K}+t_{K}-1$. Recall that $r_{K}+t_{K}<=r_{K+1}$ for a valid tower, the tower complexity is reduced overall as well.

For $s_{2}$, if it is broken but $s_{1}$ and $s_{0}$ are connected, it will also remove all the horizontal edges left to it on layer $K$ and introduce a shortcut on layer $K+1$, as shown in Fig. 4(d). In fact, all horizontal edges along $A$ will have the same behavior, and since they always remove at least one $\square$ on the next layer and only introduce one $\triangle$ on the next layer, therefore, the tower complexity keeps decreasing with $s_{1 \mid 2}$-like edges.

3) The vertical and topmost corner edges along $A$ : For $s_{3}$ on layer $K+1$, if it is broken but $s_{0 . .2}$ are connected, it will have the same effect as $S_{2}$, since $s_{3}$ is the topmost edge of a vertical path segment and there are no branches between $s_{2}$ and $s_{3}$, so the reduction is shown as $\mathcal{B} \mid S_{2 \mid 3}$ in Fig. 4(d).

4) The vertical but not topmost edges along $A$ : For $s_{4}$ on layer $K+2$, if it is broken but $s_{0 . .3}$ are connected, it will remove a $\square$ from the same layer, without introducing any $\triangle$ in any layer, as shown in Fig. 4(e). Using the tower notation, $\mathcal{T}\left(\cdots,\left(0, r_{K+2}\right), \cdots\right) \stackrel{S_{4}}{\longrightarrow} \mathcal{T}^{\prime}\left(\cdots,\left(0, r_{K+2}-1\right), \cdots\right)$. In fact, all vertical but not topmost edges along $A$ will have the same behavior, and since they always remove one $\square$ without introducing a $\triangle$, the tower complexity is further reduced.

5) The base: On the base line, serial and parallel principles can be applied to reduce the tower complexity. For example, as shown in Fig. 4(f), if layer $N$ has $t_{N} \triangle \mathrm{s}$ and $r_{N} \square \mathrm{s}$, it implies that the base layer has $b-\left(t_{N}+r_{N}\right)$ edges along a single path of connectivity $p^{b-\left(t_{N}+r_{N}\right)}$, so $P\left(\mathcal{T}\left(\cdots,\left(t_{N}, r_{N}\right), b\right)\right)=$ $p^{b-\left(t_{N}+r_{N}\right)} P\left(\mathcal{T}^{\prime}\left(\cdots,\left(t_{N}, r_{N}\right), t_{N}+r_{N}\right)\right)$ using the serial principle. For the $t_{N} \triangle \mathrm{s}$, each of them implies two parallel paths: one by the shortcut to the destination directly, and another through a horizontal edge and then a smaller tower. Since these two paths are independent (but not mutually exclusive), the PIE principle applies as $P\left(\mathcal{T}^{\prime}\left(\cdots,\left(t_{N}, r_{N}\right), t_{N}+r_{N}\right)\right)=$ $p+(1-p) P\left(\mathcal{T}^{\prime \prime}\left(\cdots,\left(t_{N}-1, r_{N}\right), t_{N}+r_{N}\right)\right)$. The PIE principle can be applied repeatedly until the base becomes $\triangle$ free. After that, another top-leftmost path $A^{\prime}$ and layer $K^{\prime}$ can identified and the above procedures can repeat to further reduce the tower complexity, until the decomposition leads to the components of known connectivity.

6) The overall recursion: As the $m * n$ lattice is a generic tower as $\mathcal{T}\left(\sqcup_{i=0}^{n-1}(0, m), m\right)$, according to Fig. 3 and Eqn. (4), $P(m, n)=P(A)+\sum_{i=0}^{m+n-1} P\left(\mathcal{B} \mid S_{i}\right) P\left(S_{i}\right)$, where $P\left(S_{i}\right)=$ $p^{i}(1-p)$, and $P\left(\mathcal{B} \mid S_{i}\right)$ are defined as follows

$$
\begin{aligned}
P\left(\mathcal{B} \mid S_{0}\right) & =p \cdot P(m, n-1) \\
P\left(\mathcal{B} \mid S_{1}\right) & =P\left(\mathcal{T}\left((1,0), \sqcup_{i=1}^{n-1}(0, m), m\right)\right) \\
\ldots & \\
P\left(\mathcal{B} \mid S_{m-1}\right) & =P\left(\mathcal{T}\left((m-1,0), \sqcup_{i=1}^{n-1}(0, m), m\right)\right) \\
P\left(\mathcal{B} \mid S_{m}\right) & =P\left(\mathcal{T}\left((m-1,0), \sqcup_{i=1}^{n-1}(0, m), m\right)\right) \\
P\left(\mathcal{B} \mid S_{m+1}\right) & =P\left(\mathcal{T}\left((m-1,0),(0, m-1), \sqcup_{i=2}^{n-1}(0, m), m\right)\right) \\
\cdots & \\
P\left(\mathcal{B} \mid S_{m+n-2}\right) & =P\left(\mathcal{T}\left((m-1,0), \sqcup_{i=2}^{n-2}(0, m-1),(0, m), m\right)\right) \\
P\left(\mathcal{B} \mid S_{m+n-1}\right) & =P\left(\mathcal{T}\left((m-1,0), \sqcup_{i=2}^{n-1}(0, m-1), m\right)\right)
\end{aligned}
$$

with the recursion rules listed above to reduce the towers to those of less complexity and the termination conditions given in Section III-A and III-B. Note that the tower complexity is always reducing by each recursion, the entire decomposition process will terminate for sure, and then the components can be reassembled, as well as the connectivity back to $P(m, n)$.

\section{Performance Evaluation}

In this section, we first offer the time complexity of the proposed approach, verify the results of lower-order lattices with other approaches symbolically to ensure the correctness, and then give more results, both by calculation and simulation, for the connectivity of higher-order lattices. The application of these results will be discussed in the next section.

\section{A. Computational Complexity}

As mentioned in the last section, one existing approach to solve the lattice connectivity problem is to use the PIE principle. $P(x, y)$ can be obtained by enumerating all possible source-destination paths (i.e., $\left(\begin{array}{c}m+n \\ n\end{array}\right)$ paths of $m+n$ segments each), crosschecking their overlapping segments, and calculating the probabilities for each combination of them. The total 
number of path combinations to examine is

$$
\sum_{i=1}^{\left(\begin{array}{c}
m+n \\
n
\end{array}\right)}\left(\begin{array}{c}
m+n \\
n \\
i
\end{array}\right)=22^{\left(\begin{array}{c}
m+n \\
n
\end{array}\right)}-1 .
$$

Because the source-destination connectivity on a lattice is symmetric along the diagonal, i.e., $P(x, y)=P(y, x)$, the total complexity of the PIE approach is $O\left(2^{\left(\begin{array}{c}m+n \\ n\end{array}\right)-1}\right)$.

In the newly proposed approach, the $m * n$ lattice is decomposed into towers. Each tower is further decomposed into towers of smaller scales. Thus the total complexity of our approach is the number of all the components generated from the decomposition process. According to the definition of Tower, it is created by converting $\square$ blocks into $\triangle$ blocks gradually from left to right and top to bottom, and depending on the number of consecutive $\square \mathrm{s}$ and $\triangle \mathrm{s}$ on each layer, there are $m \cdot n$ different configurations, since $\triangle$ blocks have to sit on top of $\square$ blocks on the next layer, and $\square$ blocks always on top of $\square$ blocks. For an $m * n$ lattice, there are at most $\left(\begin{array}{c}m+n \\ n\end{array}\right)$ paths from the source to the destination. Thus the decomposition will create at most $m \cdot n$ components starting from any top-leftmost path, in a total complexity of $O\left(m \cdot n \cdot\left(\begin{array}{c}m+n \\ n\end{array}\right)\right)$.

Although the new approach still suffers the combinatorial factor, it is much more effective than the PIE approach that has the combinatorial on the exponent. We believe that the new approach is viable, especially in many engineering scenarios where $n$ is limited, even though $m$ can grow to a large number, e.g., in a VANET city block scenario with given traffic flow directions. In addition, due to the recursive approach, when we obtain $P(m, n)$, we have also obtained all $P(x, y)$ for $x<=m$ and $y<=n$ as a byproduct, so the complexity should be amortized over all $m \cdot n$ lattices. Further, during the recursion process, the connectivity of known components can be stored for look up in new decomposition branches, which will greatly reduce the recursion depth and running time.

\section{B. Symbolic Verification}

1) $2 D$ Ladders: In [8], we solved the connectivity for 2D ladders, which is the connectivity from $(0,0)$ to $(x, 1)$ on lattices, using another decomposition approach that is not extensible to lattices of more than one layer. However, we can use that approach to verify the new one. According to [8], the following recursive expressions can be defined for $P(x, 1)$

$$
\begin{aligned}
P(x, 1) & =p\left[p^{x}+P(x-1,1)-p^{x} \theta(x)\right], x \geq 1 \\
\theta(x) & =p[p+\theta(x-1)-p \theta(x-1)], x \geq 1
\end{aligned}
$$

with $P(0,1)=p$ and $\theta(0)=0$. By simplifying these recursions, we can obtain the symbolic, non-recursion expression of the 2D ladder connectivity as follows

$$
\begin{aligned}
P(x, 1)= & \left(p ^ { x + 1 } \left(-p^{x+3}(1-p)^{x+1}\right.\right. \\
& -p(p((p-2) x+p-3) \\
& +2(x+1))+x+1)) /((p-1) p+1)^{2} .
\end{aligned}
$$

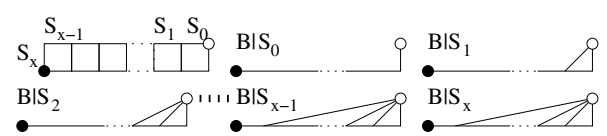

Fig. 5: The decomposition of a Ladder.

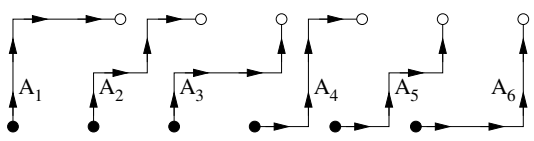

Fig. 6: All source-destination paths of a $2 * 2$ lattice.

With the new approach, as shown in Fig. 5 for illustration purposes, we have the following recursions according to the decomposition process of towers (essentially of $\triangle \mathrm{s}$ )

$$
\begin{aligned}
P(\mathcal{T}((i, 0), x))= & p^{x-i} \cdot P(\mathcal{T}((i, 0), i)) \\
P(\mathcal{T}((i, 0), i))= & p+p \cdot P(\mathcal{T}((i-1,0), i-1)) \\
& -p^{2} \cdot P(\mathcal{T}((i-1,0), i-1))
\end{aligned}
$$

with $P(\mathcal{T}((1,0), 1))=P_{T}=p+p^{2}-p^{3}$. Then with the total probability in the new approach,

$$
\begin{aligned}
P(x, 1)= & p^{x+1}+\sum_{i=0}^{x-1} P(\mathcal{T}((i, 0), x)) p^{i}(1-p) \\
& +P(\mathcal{T}((x-1,0), x)) p^{x}(1-p)
\end{aligned}
$$

which comes to the same expression as (6). For example, $P(0,1)=p$ obviously, $P(1,1)=2 p^{2}-p^{4}$, the same as that obtained in Section III-A using PIE, and

$$
\begin{aligned}
P(2,1) & =p^{7}-p^{6}-2 p^{5}+3 p^{3} \\
P(3,1) & =-p^{10}+2 p^{9}+p^{8}-2 p^{7}-3 p^{6}+4 p^{4} \\
P(4,1) & =p^{13}-3 p^{12}+p^{11}+3 p^{10}+p^{9}-3 p^{8}-4 p^{7}+5 p^{5} \\
P(5,1) & =-p^{16}+4 p^{15}-4 p^{14}-2 p^{13}+2 p^{12}+4 p^{11}+p^{10} \\
& \quad-4 p^{9}-5 p^{8}+6 p^{6} \\
& \ldots
\end{aligned}
$$

2) $2 * 2$ Lattices: Because the approach used in [8] is not capable for lattices of more than one layer, we have to use the PIE principle. Here we use a $2 * 2$ lattice as an example. To use the PIE principle, we first need to identify all the paths from the source to destination, and for the case of a $2 * 2$ lattice, there are 6 paths in total, as shown in Fig. 6.

Using the PIE principle,

$$
\begin{aligned}
P(2,2) & =P\left(A_{1}+A_{2}+A_{3}+A_{4}+A_{5}+A_{6}\right) \\
& =\sum_{k=1}^{6}(-1)^{k+1}\left(\sum_{1 \leq i_{1}<\cdots<i_{k} \leq 6}\left(A_{i_{1}} \cdots A_{i_{k}}\right)\right) \\
& =p^{12}-p^{11}+2 p^{10}+4 p^{9}+2 p^{8}-4 p^{7}-6 p^{6}+6 p^{4}
\end{aligned}
$$

where $(-1)^{k+1}$ indicates the inclusion and exclusion. Simplifying (9), we obtain the same result as that in Section III-B with the new approach.

\section{Numerical Results}

For ladders of more than one layer, or high-order lattices, however, there are no symbolic results in the literature, and the PIE complexity grows extremely quickly due to the combinatorial factor on the exponent. Thus we have to rely on the numerical results by simulation to verify the new approach. 


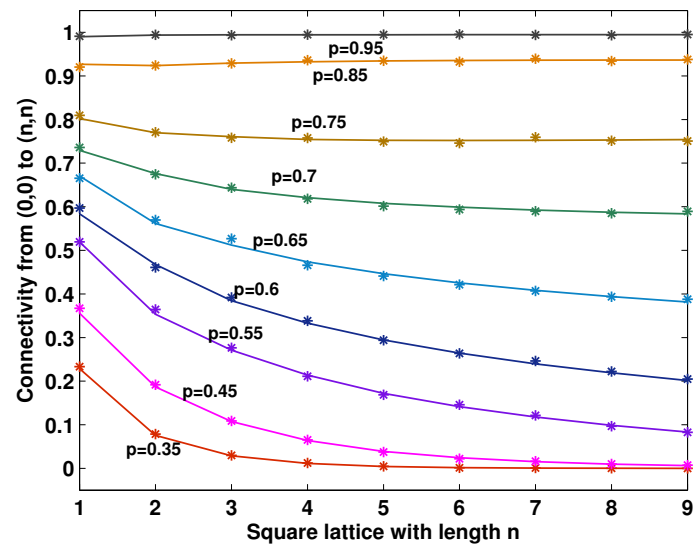

Fig. 7: The connectivity of $n * n$ lattices.

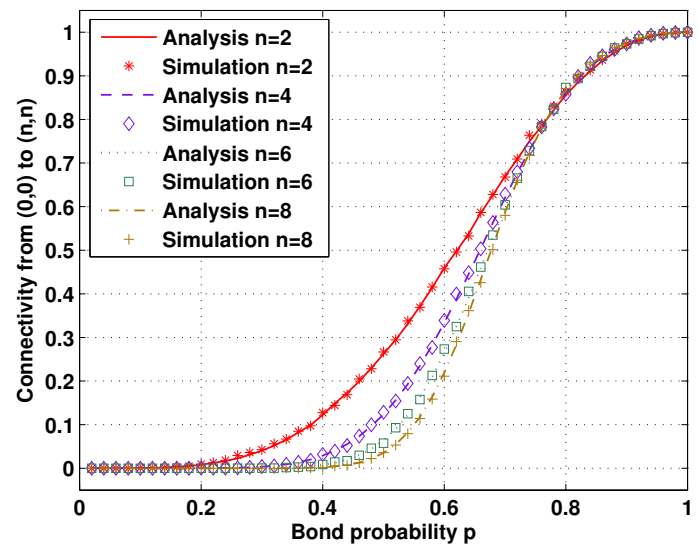

Fig. 8: The effect of bond probability on $n * n$ lattices.

1) $n * n$ Lattices: Figure 7 shows the connectivity of $n * n$ lattices. Different bond probabilities have been used from $p=$ 0.35 to 0.95 . In this and all the following figures, the lines indicate the calculation results by our new analytical approach, and the points show the results from the simulation. As we can see, the new approach produces very accurate numerical results, which have a very good match with the simulation results, but without any lengthy simulation.

We can observe that the larger the bond probability, the higher the connectivity, which is intuitive. However, the impact of bond probability is not linear. When $p$ is small, e.g., increasing $p$ from 0.35 to 0.45 , it only increases the connectivity for small $n * n$ lattices (e.g., $n<3$ ), and the increase diminishes very quickly for larger $n$. However, when $p$ is reasonably large, e.g., increasing from 0.55 to 0.65 , even though the end-toend connectivity still decreases with a large $n$, the increase due to an increased $p$ actually amplifies as $n$ increases. When $p$ is further increased, e.g., from 0.65 to 0.75 , we can see that the end-to-end connectivities are no longer to decrease with $n$ (more obviously when $p=0.85$ or 0.95 ). Recall that percolation occurs around $p=0.6447$ on an infinite lattice, the end-to-end connectivity on a finite lattice also shows the deepest gradient when $p$ is around 0.65 , illustrated by the gap between curves of different bond probabilities in Fig. 7 .
Figure 8 provides another viewpoint to the impact of the bond probability on the lattice connectivity. Larger bond probabilities always achieve higher connectivity. However, the increase is not obvious when the bond probability is less than 0.4 or above 0.8 , but the increase is very significant when it is between 0.4 and 0.8 , which means that a slight increase of the bond probability within this range will greatly increase the connectivity over lattices. Again, this corresponds to the percolation on an infinite lattice, but here we have more microscopic results on the connectivity to any vertexes on the lattice. When $n$ increases, we can see the transition from low connectivity to high connectivity becomes sharper. It is even possible to have higher connectivity for larger lattices when the bond probability is large enough, e.g., $p>0.8$, although for most small $p$, smaller lattices have higher connectivity.

2) $m * n$ Lattices: In many engineering fields (e.g., VANET in a city block scenario), we are more interested in propagating messages along certain directions (or traffic flows). In this sense, we shall focus more on the lattices with certain height. Figure 9 shows the connectivity of lattices with different heights $(n)$, when $n=2,4$ and 6 as examples. With the increase of the lattice length $m$, we can observe that the larger the bond probability, the higher connectivity. For each bond probability, the connectivity first increases, followed by an eventual decrease. The higher the lattice height, the further the peak will occur. These non-monotonic curves are very interesting to observe and very important in engineering fields to determine the optimal $m, n$ and $p$ for given applications. It shows that there is a trade-off between the total number of available paths and the length of each path. For a lattice with the given height, when the lattice length increases, the number of paths will increase, which brings more possibilities of connections between the source and destination. However, the length of these extra paths is increased too, leading to a lower probability to connect the source and destination along each path. For the overall end-to-end connectivity, path diversity has a positive effect while the path length increase has a negative one. Considering the curves shown in the figure, before the peak occurs, the positive effect of path diversity is stronger than the negative one of path length increase, leading to the increase of connectivity. However, after the peak occurs, the negative effect of path length increase becomes dominating, which leads to the decrease of the overall connectivity. The peak occurs around the cases where the lattice length equals the height, which implies a symmetric $n * n$ lattice.

Similar to $n * n$ lattices, we can offer another viewpoint to the impact of the bond probability on different $m * n$ lattices, as shown in Fig. 10. As mentioned, with the increase of lattice length, a peak connectivity will appear. The higher the lattice, the lower the peak value is. This is also because the higher the lattice, the longer the paths are. Also the peak shifts among lattices of different heights. The higher the lattices, the maximum connectivity occurs at a further distance from the source. For different bond probabilities, the decrease of connectivity after the peak grows quickly when the bond probability is larger. Nevertheless, in all figures in this section, the numerical results 


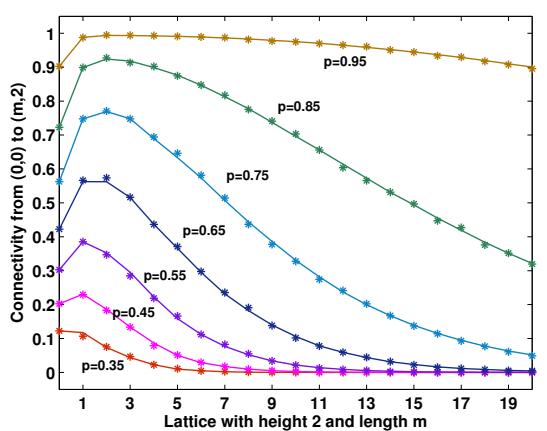

(a) Lattice with $n=2$

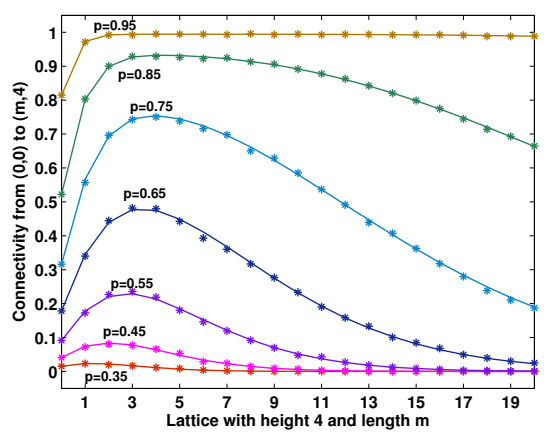

(b) Lattice with $n=4$

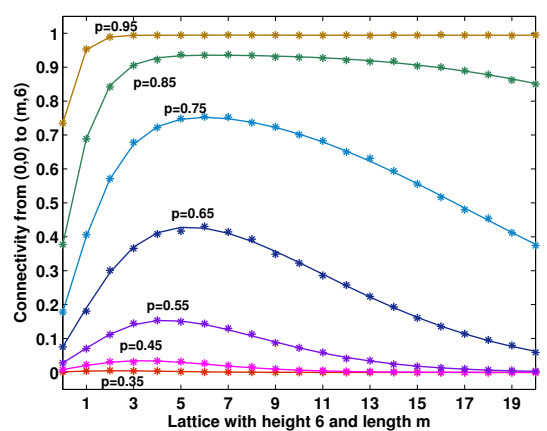

(c) Lattice with $n=6$

Fig. 9: The connectivity of $m * n$ lattices.

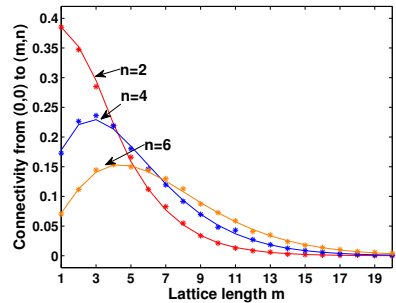

(a) $p=0.55$

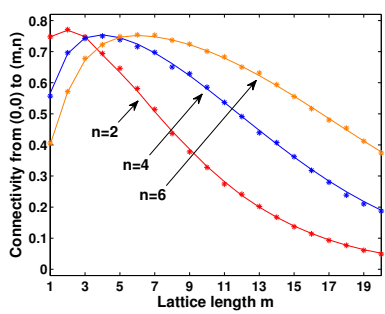

(c) $p=0.75$

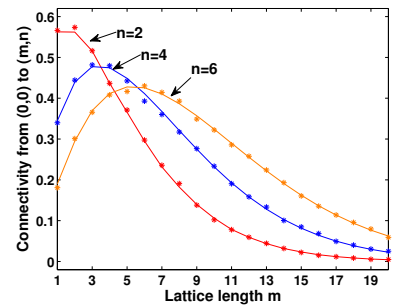

(b) $p=0.65$

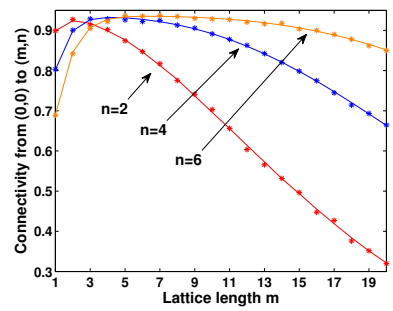

(d) $p=0.85$
Fig. 10: The effect of bond probability on $m * n$ lattices.

from the new approach are very accurate when compared with the simulation ones without lengthy simulation. In addition, the analytical expression obtained from the decomposition process can be used for further manipulation, e.g., derivatives, probability distribution functions and higher-order moments. Due to the page limit, we did not list them here.

\section{FURTHER Discussion}

\section{A. Applications in VANET}

As a motivating example, consider a VANET deployed among the vehicles traveling in a downtown area, where the road grid can be adequately modeled as a square lattice. For simplicity, we assume a homogeneous network of vehicle density $\lambda$ (in terms of the number of vehicles per unit distance) on the road. Later we will discuss the impact of heterogeneous densities. According to traffic density, speed and flow law, $\lambda$ also affects the vehicle speed, as vehicles travel slower on congested roads. A certain percentage of vehicles are equipped with communication devices and can communicate with each other (e.g., through the IEEE 802.11p-based WAVE technology in DSRC channels [27]), and the transmission range $r$ is determined by the transmission power and the channel propagation model. In order to deliver a message to a certain location in the downtown area, vehicles need to determine the optimal transmission power. If too low, the message may "die" between intersections if a vehicle cannot reach the other over the air; if too high, the probability to reach the next vehicle is higher, but it also introduces more interference and collisions with other message propagation, which can eventually reduce the probability to reach the destination given the constraints of transmission attempts, buffer size and message lifetime.

The probability for a message to reach the preceding intersection from the current one is denoted as $p$. The detailed derivation of $p$ can be referred to [8] and the derivation is based on a well-accepted assumption that the vehicle arrival follows a Poisson process. As demonstrated in that paper, $p$ can be determined by $r$, the distance $d$ between neighbor intersections (i.e., the block size), and $\lambda_{e}=\rho \lambda$, where $\rho$ is the VANET penetration rate, yielding the effective density. When considering practical issues over signal transmission, e.g., signal interference and shadowing, a shadowing factor is also introduced into the derivation of $p$. Once we have $p$, a lot of VANET properties can be explored, previously by simulation in [8], and now with the analytical expressions obtained by the new approach.

Depending on the nature of a message, the message may want to be propagated among the same direction as the vehicle (e.g., emergency vehicles), which is equivalent to the onedimensional connectivity $P(x, 0)$ on lattices. If the message wants to be propagated among the same street and $n$ neighbor ones in the same direction (e.g., congestion notification), twodimensional ladder connectivity $P(x, n)$ can be used. Further, if the message can be disseminated to all intersections in the downtown area (e.g., parking availability), lattice connectivity $P(x, y)$ for all $x<=m$ and $y<=n$ applies. As shown in Section IV-C, being close to the source does not necessary mean the message is more likely to be received-it also 
depends on the constraints on the propagation directions. Also, increasing the transmission power will have different effect on the connectivity. For example, when the connectivity is low, slightly increasing the transmission power does not have much effect beyond a few intersections, until the transmission power is sufficiently high as shown in Fig. 9, where the connectivity can be considerably improved. Above that, further increasing the transmission power will not improve connectivity much, but on the other hand will introduce more interference and collisions. Thus the optimal transmission power tuning also depends on the intended distance and reliability of the message propagation, and the results presented in this paper, as well as the new approach to obtain these results, can be of great value in practice for a range of applications.

\section{B. Limitations and Extensions}

In this paper, we illustrated the new decomposition approach following the mutually exclusive events and total probability with square lattices and the homogeneous bond probability. However, the approach itself is not limited by the size and shape of the lattice, as well as the bond probability on each edge. The key insights are the introduction of mutually exclusive events in the decomposition of the lattice, and the way being able to decompose all components recursively. It is our intention to apply the same technique to other directed connectivity problems on other regular tiling lattices, including triangles and hexagons used in many applications.

On the other hand, the new approach still encounters a combinatorial factor in the polynomial sense (which is much better than the PIE principle that has the combinatorial factor on the exponent), as there indeed exist $\left(\begin{array}{c}m+n \\ n\end{array}\right)$ distinct paths. When both $m$ and $n$ are large, this will lead to a very large decomposition space. Dynamic programming approaches can be used to leverage the known connectivity of smaller components, but when the analytical expressions are reassembled, it will lead to extremely high-order polynomials (the order is as high as $2 m n+m+n)$. Luckily, in most engineering problems, one dimension is often of limited size while the other dimension can grow, which keeps the combinatorial factor manageable. Also depending on the needed precision, polynomial truncation can be utilized to limit the length and complexity of the analytical expressions.

Although it is our goal to shed new light on the directed percolation problem, since the polynomial grows quickly if $m$ and $n$ go to infinity, so far it is not possible for us to obtain the explicit expression for arbitrarily large $m$ and $n$. However, the parameterized recursive expressions based on $m$ and $n$ are readily available, and we are intended to explore more properties based on these recursive expressions.

\section{CONCLUSIONS}

In this paper, by proposing a new decomposition approach based on the mutually exclusive events and total probability, we presented a scheme to obtain the directed connectivity on arbitrary square lattices. The results are given in a recursive manner, and the explicit expressions can be obtained for lower-order lattices. The approach and the obtained results are validated with the existing approaches and numerical results, which confirm the correctness of the new approach and the accuracy of the analytical results without lengthy simulation.

\section{REFERENCES}

[1] M. Desai and D. Manjunath, "On the connectivity in finite ad hoc networks," IEEE Comm Letters, 6(10):437-439, 2002.

[2] O. Dousse, P. Thiran, and M. Hasler, "Connectivity in ad-hoc and hybrid networks," in Proc. IEEE INFOCOM'02, pp. 1079-1088, 2002.

[3] H. Nishiyama, T. Ngo, N. Ansari, and N. Kato, "On minimizing the impact of mobility on topology control in mobile ad hoc networks," IEEE Trans. on Wireless Commu., 11(3):1158-1166, 2012.

[4] S. He, J. Chen, and Y. Sun, "Coverage and connectivity in duty-cycled wireless sensor network for event monitoring," IEEE Trans. on Parallel and Distributed System, 23(3):475-482, 2012.

[5] K. Miyao, H. Nakayama, N. Ansari, and N. Kato, "LTRT: an efficient and reliable topology control algorithm for ad-hoc networks," IEEE Trans. on Wireless Commu., 8(12):6050-6058, 2009.

[6] S. Ukkusuri and L. Du, "Geometric connectivity of vehicular ad hoc networks: Analytical characterization," Transportation Research Part C: Emerging Technologies, 16(5):615-634, 2008

[7] S. Shioda, J. Harada, Y. Watanabe, T. Goi, H. Okada, and K. Mase "Fundamental characteristics of connectivity in vehicular ad hoc networks," in Proc. IEEE PIMRC'08, pp. 1-6, 2008.

[8] Y. Zhuang, J. Pan, and L. Cai, "A probabilistic model for message propagation in two-dimensional vehicular ad-hoc networks," in Proc. ACM VANET'10, pp. 31-40, 2010.

[9] A. M. Mathai, "An introduction to geometrical probability: distributional aspects with applications," Gordon and Breach Science, 1999.

[10] F. Baccelli and B. Blaszczyszyn, "Stochastic geometry and wireless networks," NOW Publishers, 2009.

[11] C. Kurrer and K. Schulten, "Dependence of percolation thresholds on lattice connectivity," Phys. Rev., E 48, pp. 614-617, 1993.

[12] G. Grimmett, "Percolation," 2nd Ed, Springer, 1999.

[13] L. Chen and F. Wu, "Directed percolation in two dimensions: An exact solution," Differential Geometry and Physics, Nankai Tracts in Math., 10:160-168, 2006.

[14] Percolation threshold, available at http://en.wikipedia.org/wiki/ Percolation_threshold, accessed in 2012.

[15] L. Chen, "Asymptotic behavior for a version of directed percolation on a square lattice," Physica A, 390(3):419-426, 2011.

[16] H. Cai, X. Jia, and M. Sha, "Critical sensor density for partial connectivity in large area wireless sensor networks," in Proc. IEEE INFOCOM'10, 2010.

[17] J. Gao and L. Guibas, "Geometric algorithms for sensor networks," Philosophical Trans. of the Royal Society A, 370(1958):27-51, 2012.

[18] P. Wan, K. Alzoubi, and O. Frieder, "Distributed construction of connected dominating set in wireless ad hoc networks," in Proc. IEEE INFOCOM'02, pp. 1597-1604, 2002.

[19] Y. Zhuang and J. Pan, "Random distances associated with equilateral triangles," arXiv:1207.1511, 2012.

[20] Y. Zhuang and J. Pan, "Random distances associated with rhombuses," arXiv:1106.1257, 2011.

[21] Y. Zhuang and J. Pan, "Random distances associated with hexagons," arXiv:1106.2200, 2011.

[22] M. Haenggi, J. Andrews, F. Baccelli, O. Dousse and M. Franceschetti, "Stochastic geometry and random graphs for the analysis and design of wireless networks," IEEE Journal on Selected Areas in Commu., 27(7):1029-1046, 2009.

[23] I. Glauche, W. Krause, R. Sollacher, and M. Greiner, "Continuum percolation of wireless ad hoc communication networks," Physica A, 325(3):577-600, 2003.

[24] P. Santi, "Topology control in wireless ad hoc and sensor networks," ACM Computing Surveys, 37(2):164-194, 2005.

[25] D. Lu, X. Huang, P. Li, and J. Fan, "Connectivity of large-scale cognitive radio ad hoc networks," in Proc. IEEE INFOCOM'12, pp. 1260-1268, 2012.

[26] Y. Zhuang, J. Pan, Y. Luo, and L. Cai, "Time and location-critical emergency message dissemination for vehicular ad-hoc networks", IEEE Journal on Selected Areas in Commu., 29(1):187-196, 2011.

[27] http://www.leearmstrong.com/DSRC/DSRCHomeset.htm 